\title{
Mitral valve disease in sarcoidosis diagnosed by cardiovascular magnetic resonance
}

Vasiliki Tsampasian, Clint Maart, Vassilios S Vassiliou, Pankaj Garg

Department of Cardiology, Norfolk and Norwich University Hospital, Norwich, UK (V Tsampasian MSc, C Maart MD, V S Vassiliou PhD, P Garg PhD); Norwich Medical School, University of East Anglia, Norwich, UK (V S Vassiliou, P Garg)

Correspondence to:

Dr Pankaj Garg, Norwich Medical School, University of East Anglia Norwich NR4 7TJ, UK p.garg@uea.ac.uk

An 81-year-old man was referred to the clinic with a 1-year history of shortness of breath on exertion. His past medical history included cryptogenic liver cirrhosis, idiopathic pulmonary fibrosis. On examination we found him to have a pan-systolic murmur and fine bi-basal crackles on chest auscultation. Resting heart and respiratory rate were within normal range (73 and 17 respectively) and saturations were $96 \%$ on air. Laboratory investigations showed normal white blood count and Creactive protein. ECG showed sinus rhythm with first degree heart block.

An echocardiogram revealed normal left ventricular size and function and possible bi-leaflet mitral valve prolapse resulting in severe mitral regurgitation (Appendix figure 1). Cardiovascular magnetic resonance $(\mathrm{CMR})$ showed a central jet of mitral regurgitation caused by annular dilatation and noncoaptation of the mitral valve leaflets (figure); using both the standard method, defined as left ventricular stroke volume minus the aortic net flow, and 4D flow advanced techniques, the mitral regurgitation was found to be $5 \mathrm{ml}(<30 \mathrm{ml}$ is mild) (Appendix figure 2). Additionally, the mitral regurgitation jet was seen swirling around the left atrium thus leading to an overestimation of the regurgitation using a speckle-Doppler trace (figure; video). Furthermore, moderate left ventricular systolic impairment, with a non-ischaemic fibrosis pattern in the lateral and anterior walls, was seen; on T1-mapping, there was globally increased T1 signal and raised extracellular volume (figure). Taken 
together, the findings suggested non-ischaemic cardiomyopathy secondary to cardiac sarcoidosisand on discussion with the respiratory team, it became apparent that the patient had, 20 years earlier, indeed been treated abroad for lung sarcoid. We then decided to defer the initial plan of cardiac surgery on the mitral valve, as it was evident post CMR that he had a primary cardiomyopathy with only mild mitral regurgitation, and unrelated symptoms. Following review by the respiratory team, it was felt that this is a chronic process, hence close monitoring of the disease was recommended.

Multiparametric CMR imaging is invaluable not only for the accurate assessment of function and sub-phenotyping the aetiology of cardiomyopathy, but also, using 4D flow methods, for more precise quantification of mitral regurgitation that might-as shown in our case-completely change the course of clinical management.

\section{Contributors}

We all contributed to writing the manuscript. VT wrote the first draft of the manuscript. CM, VV, and PG edited the manuscript. PG obtained the images. Written consent for publication was obtained from the patient

\section{Declaration of interests}

We declare no competing interests.

(C) 2021 Elsevier Ltd. All rights reserved. 
Figure: Multiparametric cardiovascular magnetic resonance in sarcoidosis

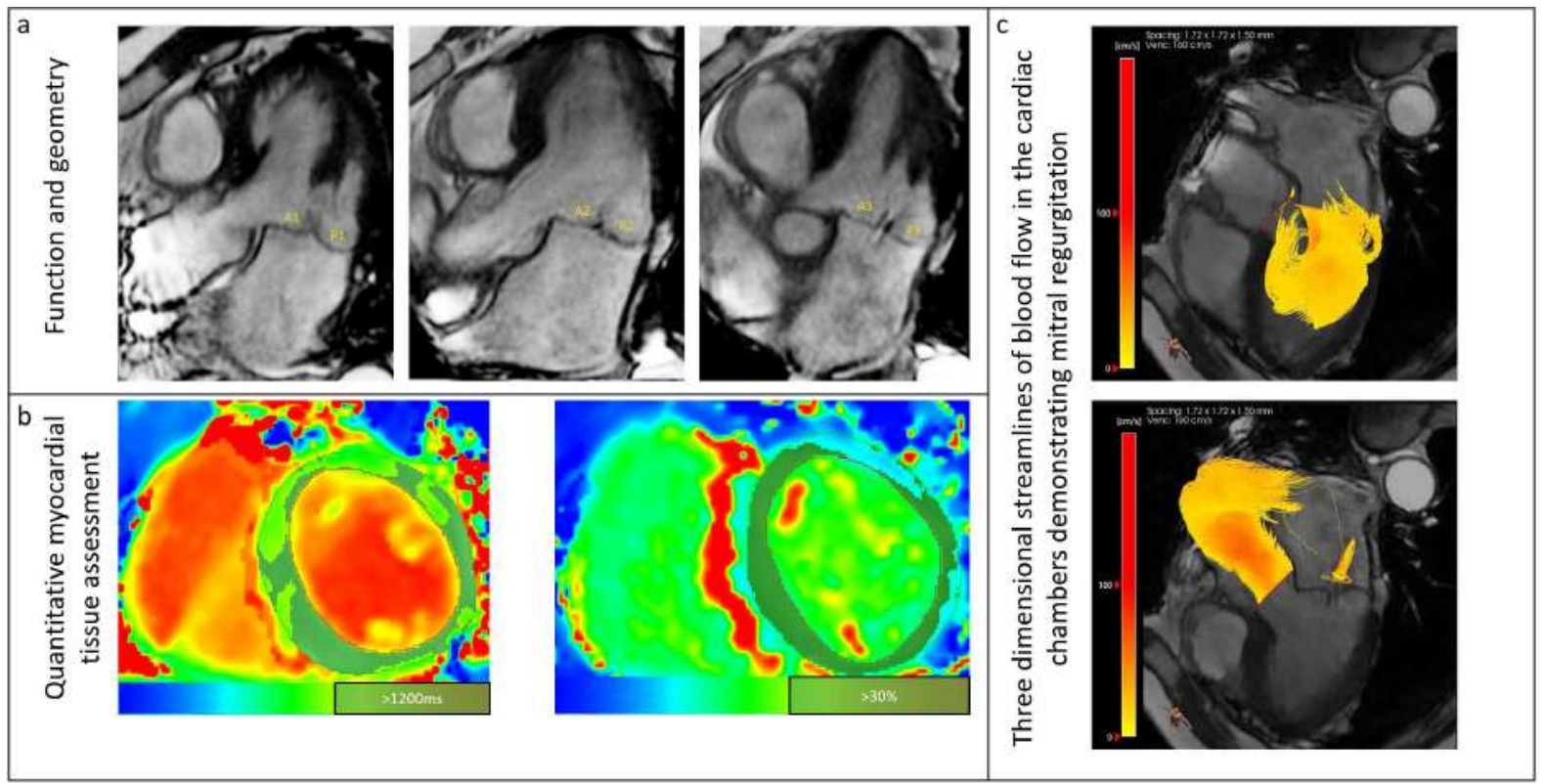

CMR assessment of the mitral valve and the left ventricle. Panel a - Central jet of mitral regurgitation because of non-coaptation of the leaflets. Panel $b-T 1$ mapping demonstrating globally increased raised extracellular volume suggestive of non-ischaemic cardiomyopathy. Panel c-Mitral regurgitation jet swirling in the left atrium as assessed by CMR.

\section{Multiple choice question}

\section{Using cardiovascular magnetic resonance to clinch the diagnosis}

An 81-year-old man presented with 1-year history of shortness of breath. Clinical examination showed a pan-systolic murmur. Look at the findings of the echocardiography and cardiovascular magnetic resonance and suggest the most likely diagnosis.
A) Mild mitral regurgitation and non-ischaemic cardiomyopathy
B) Mild mitral regurgitation and ischaemic cardiomyopathy
C) Tricuspid regurgitation
D) Aortic regurgitation

Answer A) 